\title{
Motion of liquid and stabilising particles in individual liquid aluminium alloy films
}

\author{
Korbinian Heim ${ }^{1,2}$, Alexei Ershov ${ }^{3}$, Alexander Rack ${ }^{4}$, John Banhart ${ }^{1,2}$, and Francisco García-Moreno ${ }^{1,2, *}$ (b) \\ ${ }^{1}$ Technische Universität Berlin, Hardenbergstraße 36, 10623 Berlin, Germany \\ ${ }^{2}$ Helmholtz-Zentrum Berlin für Materialien und Energie, Hahn-Meitner-Platz 1, 14109 Berlin, Germany \\ ${ }^{3}$ Karlsruher Institut für Technologie, Hermann-von-Helmholtz-Platz 1, 76344 Eggenstein-Leopoldshafen, Germany \\ ${ }^{4}$ European Synchrotron Radiation Facility, 38043 Grenoble, France
}

Received: 29 January 2020

Accepted: 25 June 2020

Published online:

8 July 2020

(C) The Author(s) 2020

\begin{abstract}
Liquid metallic films are the constituting elements of metallic foams and are governed by a complex interplay between the liquid-here aluminium alloys-, the film stabilising solid particles contained in the melt, and the gas phase. We use synchrotron X-ray radioscopy to study particle dynamics and analyse particle motions by applying novel image processing techniques. We find that particles move alone and as clusters. They may move permanently with the melt or can be trapped by the film surface and subsequently even de-trapped again. The aim of this work is to observe interactions between particles or particle clusters and the film surfaces, and to understand how liquid flow in films is reduced and film stability increased by such particles
\end{abstract}

\section{Introduction}

Foams are assemblies of various structural elements: two-dimensional curved faces (also called films) partitioning space into cells, one-dimensional socalled Plateau borders at the intersection of three films, and nodes in which 4 Plateau borders meet [1]. A liquid metal foam forms a solid structure after solidification, which is seen as a promising engineering material. Metal foams are at the best metastable due to their large interface areas and can be conserved only if the melts contain solid nonmetallic particles, which are responsible for film and foam stabilisation, dispersed in the liquid phase. If they attach to the film surface, they hinder film thinning and consequently film rupture, if they flow through not. Particles are also a marker for liquid velocity. The velocity of the liquid represents the liquid flow and e.g. the effect of capillary suction or drainage, which results in film thinning, leading to film instabilities and later rupture. The amount and fixing velocity of particles to the film surface/oxide skin is a measure for the velocity of film stabilisation. In liquid aluminium alloys, these solid particles are often silicon carbide [2]. How many of such particles are needed to keep a film stable long enough, and how large they should be has been the subject of

Address correspondence to E-mail: garcia-moreno@helmholtz-berlin.de 
optimisation studies [3]. One should keep their content as low as possible since they embrittle solid foams and make their recycling difficult.

Analyses of microstructures of solid cell walls (in films and foams) have been undertaken to know how particles are arranged within [4, 5]. However, such post-solidification studies do not reveal how particles move through liquid films and to which locations they go to keep films stable. During solidification, particles might have been pushed to different locations, which is why in situ studies on liquid systems should be carried out. Tomography on particle-containing foams in near-equilibrium has shown the location of particles without, however, revealing dynamics [6]. In situ radioscopy on individual liquid metal films has recently revealed how particles move through films right after being drawn from a liquid [7]. Furthermore, the joint action of particles and ambient oxygen on film stabilisation was demonstrated [8] and claimed to be the basis of the resistance of films to rupture [9]. Strong phase contrast in corresponding radiographic images and high repetition frequencies have made this possible. One important observation in these studies has been that particles or particle clusters can behave in three different ways: some flow continuously through a film or Plateau border (type 1), others remain at a fixed position from the very beginning of film drawing (type 2), while a third type of particles first flows but then is stopped at a certain instant and does not move any further (type 3) [7]. Some of these observations were based on quantitative image analysis, and others on a qualitative assessment based on manual inspection of individual events observed in radiographic image sequences.

The overall objective of this work is to understand the dynamics of particle and melt flow in liquid metal films and to highlight their role in stabilising metal foams. Furthermore we quantify particle and liquid velocity in the vertical and horizontal direction to find out how gravity-induced drainage and capillary suction influence film thickness and study how particle clustering and interactions with the oxide layer contribute to film (and eventually foam) stabilisation. Synchrotron X-ray radioscopy combined with advanced image analysis tools are applied to gain quantitative information and to derive new conclusions from particle flow.

\section{Materials and methods}

An AlSi9Mg0.6 alloy (all values in wt $\%$ ) reinforced with $20 \mathrm{vol} \% \mathrm{SiC}$ particles of $10 \mu \mathrm{m}$ mean particle size made by Alcan, Montréal, Canada, was used (ANSI H35.5 designation: AlSi9Mg0.6/SiC/20p). Experiments were carried out in a closed chamber under an air/argon mixture containing 1500 ppm oxygen or in air. The oxygen content has an influence on foam and film stabilisation as we know from earlier work $[7,8]$, but in the present paper this is a side aspect. To melt the composite, two vertically arranged parallel ceramic heating plates were used to heat up a crucible placed in between and to keep it at $680{ }^{\circ} \mathrm{C}$ [8]. The alumina crucible $(\Phi=30 \mathrm{~mm}$, $h=40 \mathrm{~mm}$ ) was filled up to the brim with $\approx 80 \mathrm{~g}$ of melt. To guarantee an optimal distribution of particles, the melt was stirred before every individual experiment.

Metallic films were made by dipping a set of two wire frames arranged in parallel (so-called quadruple parallel frame) into a melt, pulling it out after and stopping after the upper pair had reached the field of view (FOV) of X-ray imaging, see Fig. 1a [10]. The lower frame pair was kept in the melt, while the liquid film was suspended between the upper two wire frames (Fig. 1b). The shape of such films then resembles that of a Plateau border in foams. Due to good thermal flow from the melt via a continuous bridge of liquid along the connecting wires, the upper film remained liquid. The lower frame pair serves as a dummy, its shape not being relevant as only the upper frame pair shapes the liquid and forms an artificial film and a Plateau border as shown in Fig. 1c [11]. The moment the measurements start is the moment at which the film comes to a rest after pulling, which takes $\approx 2 \mathrm{~s}$. This defines zero time. Films were kept liquid for $30 \mathrm{~s}$ to study drainage and particle movements until the film solidified, see an example in Fig. 1d.

In situ radioscopy experiments were carried out using hard synchrotron radiation available at the ESRF beamline ID19 (Grenoble, France) [12]. High imaging sensitivity in terms of phase contrast and a sufficient photon flux density for short acquisition times were achieved by operating the beamline opticfree: only a diamond absorber in the front end of the beamline and the mandatory beryllium window were placed in the X-ray optical beam path. This, in combination with the 150-m long beamline, ensures 
(a)

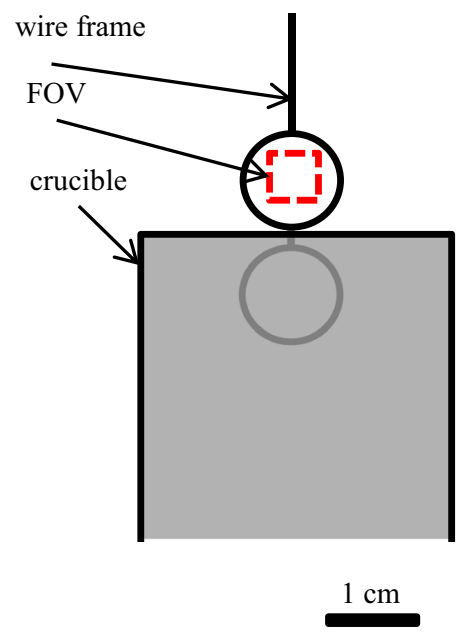

(b)

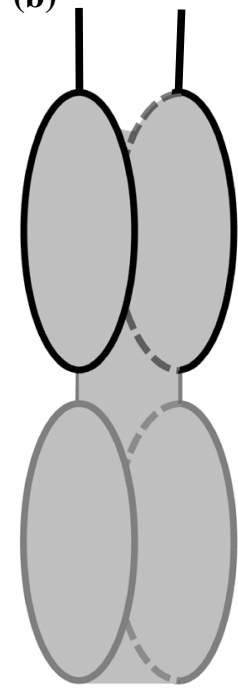

(c)

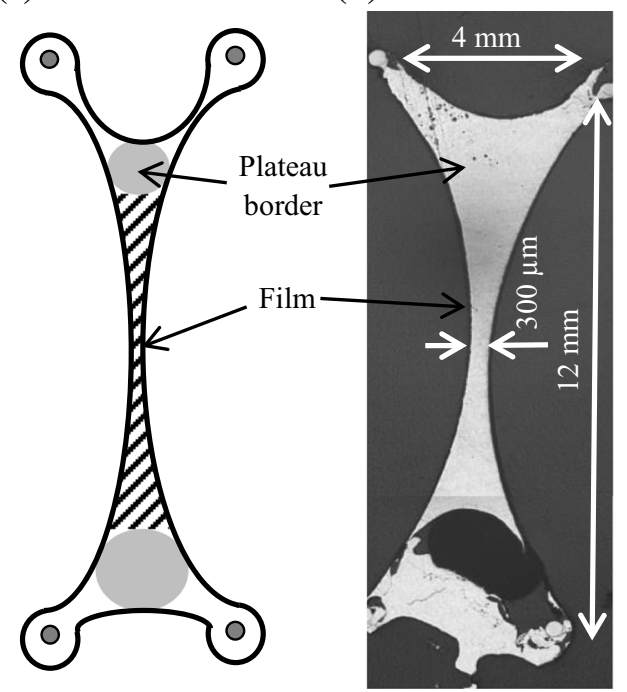

Figure 1 a Schematic of the film pulling process and the thermally coupled quadruple parallel frame arrangement. The red broken frame indicates the field of view (FOV) for synchrotron radioscopy into which the film was transferred while still liquid. b Perspective schematic of frame configuration in a, where the

excellent phase contrast. The beamline's so-called single-harmonic undulator U17.6 was utilised as a source (peak photon energy $\sim 19 \mathrm{keV}$ ). For phase contrast imaging, the detector was positioned approximately $1 \mathrm{~m}$ downstream of the sample. The former consisted of a $47-\mu \mathrm{m}$ thick GGG:Eu singlecrystal scintillator (Eu-doped $\mathrm{Gd}_{3} \mathrm{Ga}_{5} \mathrm{O}_{12}$ ) on top of a $500-\mu \mathrm{m}$ thick undoped GGG substrate. The luminescence image was projected via a mirror and a $4 \times /$ $0.2 \mathrm{NA}$ objective (OptiquePeter, Lentilly, France) onto the sensor of a pco.dimax camera $(2016 \times 2016$ pixels, $11 \mu \mathrm{m}$ sensor pixel size, up to 1200 frames/s recording rate, PCO AG, Kehlheim, Germany). The effective pixel size of the complete detection system was $2.7 \mu \mathrm{m}$. Images were recorded at a frame rate of $50 \mathrm{~Hz}$, which was found to be sufficient for our purpose.

Data processing starts with the equalization of nonuniform brightness variations. For each image frame, we subtracted a low-pass filtered version of the original image. This step removes varying large-scale brightness patterns but retains local details and was implemented using publicly available ImageJ/Fiji software [13]. The SiC particles were also used for tracking purposes, e.g. to evaluate the velocity of the draining liquid melt. In order to compute quantitative characteristics of particle and liquid motions, we lower shaded frames are dummy frames. c, d Side view crosssection of the relevant upper wire frame pair and the corresponding suspended metallic film (schematic and optical microscopic image after solidification).

employed optical flow methods. The optical flow model takes into account multiple image features and employs spatio-temporal smoothness. More details about the model can be found elsewhere [14, 15]. As a result of optical flow computations, we obtained a dense flow field, which can be further analysed (e.g. by computation of magnitude, direction, etc.).

During some of the quantification steps, particles were eroded by $1-2$ pixels (corresponding to $2.7-5.4 \mu \mathrm{m}$, respectively), for example for image segmentation. As the $\mathrm{SiC}$ particle size is nominally $10 \mu \mathrm{m}$, the information lost due to elimination of smaller particles is negligible, especially because the most pronounced effects are observed for large particles and particle clusters. Temporally colour-coded particle trajectories are obtained by particle tracking and temporal colour integration over $4 \mathrm{~s}$.

\section{Results and discussion}

\section{General overview}

To give a first overview, particles permanently in motion (type 1 as defined in the introduction) in an AlSi9Mg0.6/SiC/20p film are highlighted by temporally colour-coded particle trajectories (Fig. 2). Particles that remained fixed throughout the experiment 
(type 2) were removed. Background correction, integration over the minimum intensity value and colouration were performed to plot the path of the particles. Each colour stands for the time at which a single frame was taken. This simple visualization technique already makes the complexity of particle movements evident. Both downward and horizontal particle motions can be observed, which are caused by gravity forces and capillary suction into the Plateau border situated on the right edge of the images, respectively. To further analyse different aspects of liquid dynamics, several quantifying analyses were performed by image processing. Interactions of different particle types are summarised in "Direction change of particle motion" section.

\section{Quantification of moving clusters}

In Fig. 3a, the total area of moving particles in the film shown in Fig. 2 is given. The threshold value of a moving cluster or particle is set to 1.2 pixels $(3.24 \mu \mathrm{m})$. At the beginning of the analysis, most of the moving particles are present in the FOV but consecutively leave it as time elapses, i.e. they are of type 1 . This is reasonable considering the film pulling procedure: Initially, films are thick and full of particles and oxidation is at a low level and therefore particle retention is weak.

Classifying and counting the number of particles or particle clusters with respect to their size (large or small) yields Fig. 3b, c. Small clusters (here $\leq 3645 \mu \mathrm{m}^{2}$ ) represent the major category of moving clusters, compare scaling of Fig. 3b, c. A similar decay for particle numbers is observed as for the global area given in Fig. 3a. After around $25 \mathrm{~s}$ almost no big cluster is moving any more. This means that particles are either collected at the bottom (outside the FOV) or stick to the oxide layer (within the FOV). The former particles are of type 1, and the latter of type 3 . Big clusters cannot flow anymore after a certain time due to the film thickness reduction that restrains big objects immersed in the fluid film.

\section{Velocity analysis}

The average velocity of clusters as well as their horizontal $(x)$ and vertical $(y)$ components are analysed and shown in Fig. 4. The measurements show that clusters decelerate in the vertical but accelerate in the horizontal direction within the first $5 \mathrm{~s}$ (Fig. $4 \mathrm{~b}, \mathrm{c}$ ), decelerating slightly on average (Fig. 4a). Particles move to the right here due to the suction of the Plateau border situated at the right side of the image. The downward movement follows gravity forces. As the $\mathrm{SiC}$ particles flow in the melt, we can assume that they move with the velocity of the liquid.

Right after film pulling the acceleration of particles/clusters is driven mainly by gravitational forces, therefore a fast downward but only moderate movement to the right. Later, particles/clusters are pushed out of the film due to increasingly radially acting (in our FOV horizontal) capillary forces and slow down downward induced by film thinning (see Fig. $5 b$ ). This situation is similar to that in aqueous films [16].

Based on Hagen-Poiseuille flow between two immobile surfaces, the time-dependent loss of liquid (a)

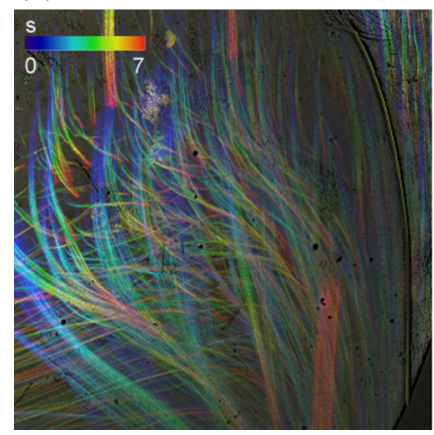

(b)

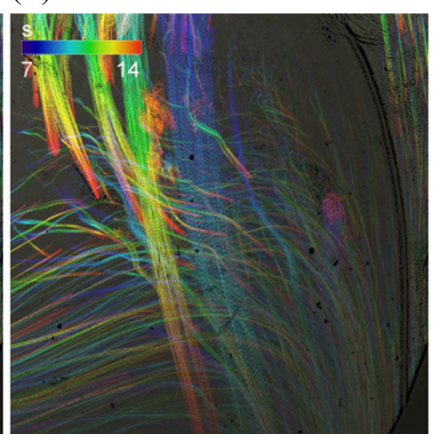

(c)

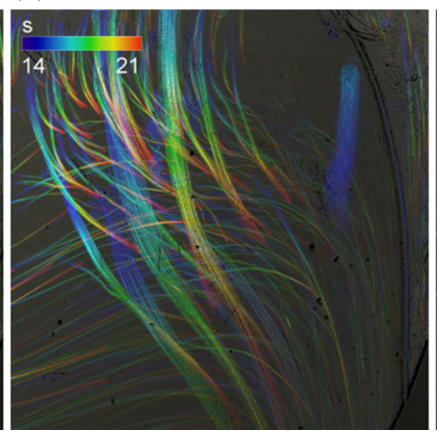

(d)

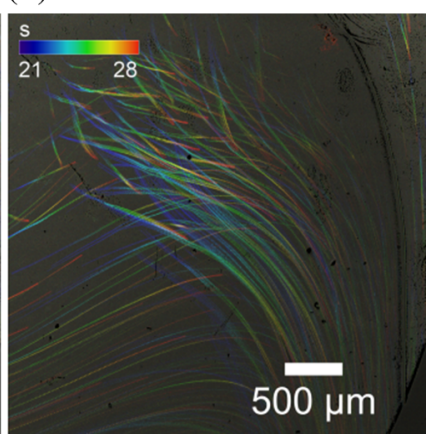

Figure 2 Trajectories of SiC particles in an AlSi9Mg0.6/SiC/20p film pulled in $1500 \mathrm{ppm} \mathrm{O}_{2}$ atmosphere from the melt held at $680^{\circ} \mathrm{C}$. The colour scale defines particle positions at a given time (in seconds) in the intervals a $0-7 \mathrm{~s}, \mathrm{~b} 7-14 \mathrm{~s}, \mathrm{c} 14-21 \mathrm{~s}$ and d 21-28 s after pulling. A vertical Plateau border is located close to the right side of the field of view. This induces preferential particle motion to the right. 


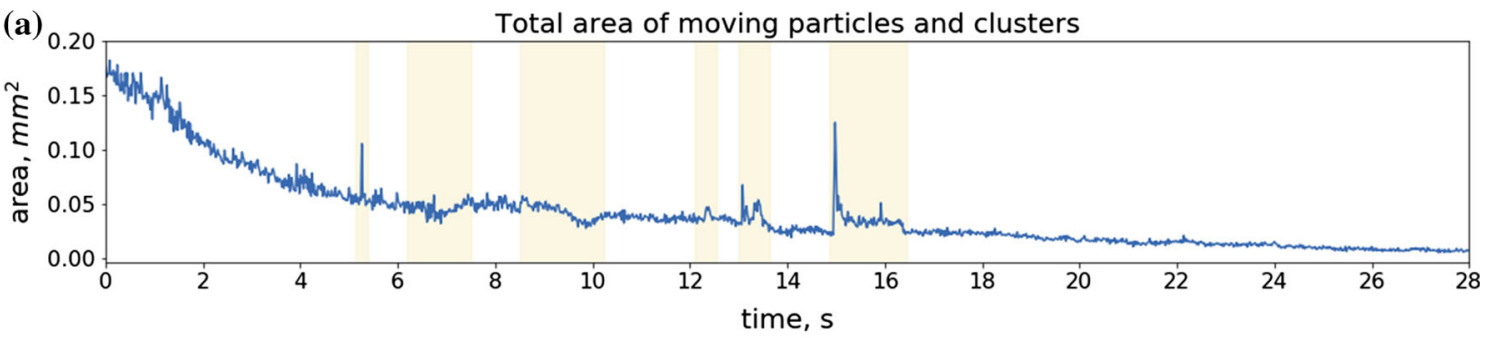

(b)

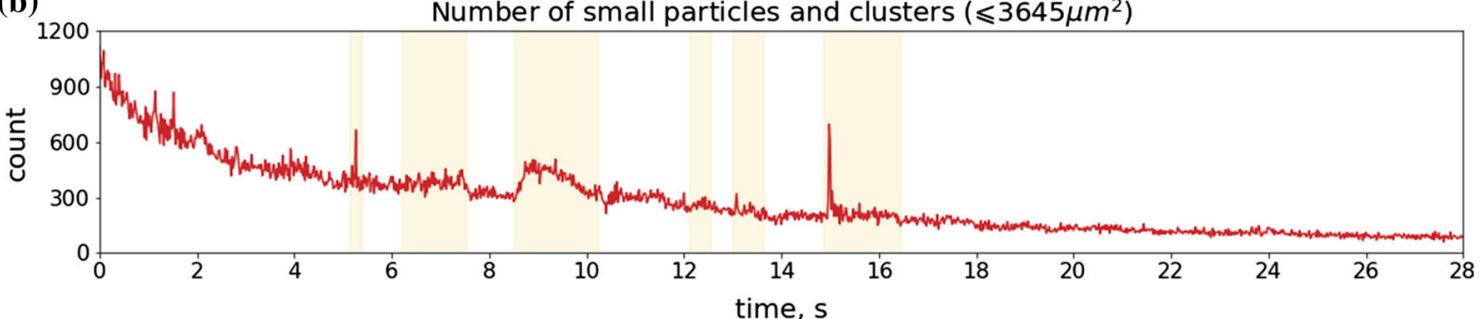

(c)

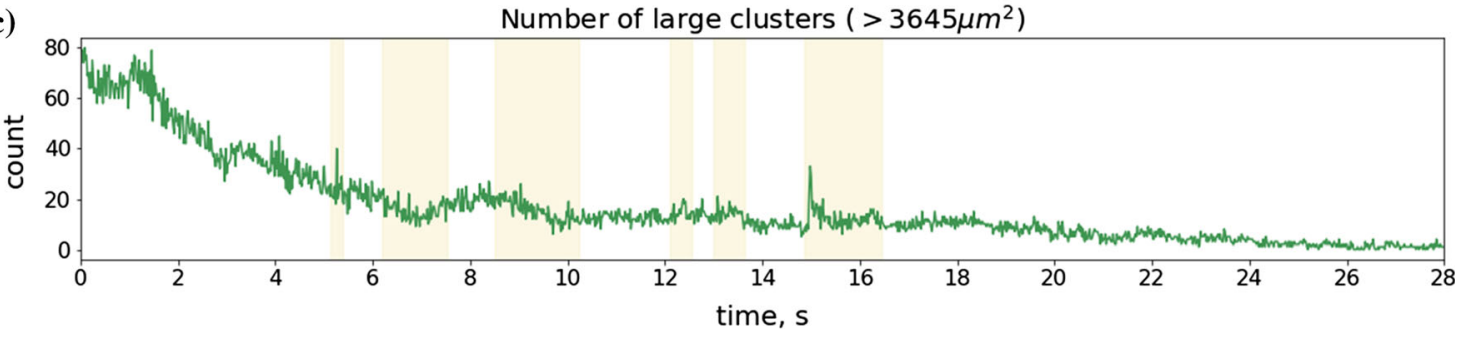

Figure 3 a Total area of moving particle clusters in a AlSi9Mg0.6/SiC/20p film pulled in $1500 \mathrm{ppm} \mathrm{O}_{2}$ atmosphere corresponding to Fig. 2. b Number of clusters smaller than

volume $V$ in a vertically arranged film can be written as $[7,17,18]$

$-\frac{\mathrm{d} V}{\mathrm{~d} t}=\frac{\operatorname{l\rho g} x^{3}}{12 \mu}$

where $x(t)$ is the time-dependent film thickness, $\rho$ is the liquid density $\left(2.42 \mathrm{~g} \mathrm{~cm}^{-3}\right.$ for AlSi9 at $700{ }^{\circ} \mathrm{C}$ [19]), $l$ is the lamella width, $\mu$ melt viscosity (3.3 $\mathrm{mPa} s$ for $\mathrm{AlSi} 9 / \mathrm{SiC} / 20 \mathrm{p}$ at $700{ }^{\circ} \mathrm{C}$ [20]), and $g=9.81 \mathrm{~m} \mathrm{~s}^{-2}$ gravity acceleration. The liquid volume in a simplified lamella is $[7,18]$

$V=\operatorname{lh} x$,

where $h$ and $l$ are the lamella height and width, respectively, corresponding here to the dimension of the selected FOV of $3.5 \times 3.5 \mathrm{~mm}$. Assuming $l$ and $h$ as constant, the time dependence of the volume is then

$\frac{\mathrm{d} V}{\mathrm{~d} t}=\ln \left(\frac{\mathrm{d} x}{\mathrm{~d} t}\right)$.
$3645 \mu \mathrm{m}^{2}$ and $\mathrm{c}$ larger than $3645 \mu^{2}$. Yellow shaded regions highlight where particle motion shows changes due to specific events explained in Fig. 10.

Substituting (2) and (3) into Eq. (1), integration of Eq. (1) and solving it for $x(t)$ results in an expression for the time-dependent film thickness: $[17,18]$

$x(t)=x_{0} \sqrt{\frac{6 \mu h}{6 \mu h+\rho g x_{0}^{2} t}}$,

where $x_{0}=4 \mu \mathrm{m}$ is the starting lamella thickness during film pulling after $t=-2 \mathrm{~s}$ [7]. Furthermore, and assuming that no liquid flows out of the FOV horizontally, what is approximately the case at $t=0 \mathrm{~s}$ (see Fig. 4b), the vertical velocity of the liquid can be expressed as

$\frac{\mathrm{d} y}{\mathrm{~d} t}=\frac{h}{x_{0}} \frac{\mathrm{d} x}{\mathrm{~d} t}$.

This simple model results in an extremely fast film thinning with the given parameters (see Fig. 5a) [7]. Accordingly, the film should have already thinned down to $x=37 \mu \mathrm{m}$ at $t=0 \mathrm{~s}(2 \mathrm{~s}$ after film pulling, when the measurement starts). However, the reported minimum film thickness is $x_{\min } \approx 50 \mu \mathrm{m}$ $[5,21,22]$ and the measured film thickness of the 

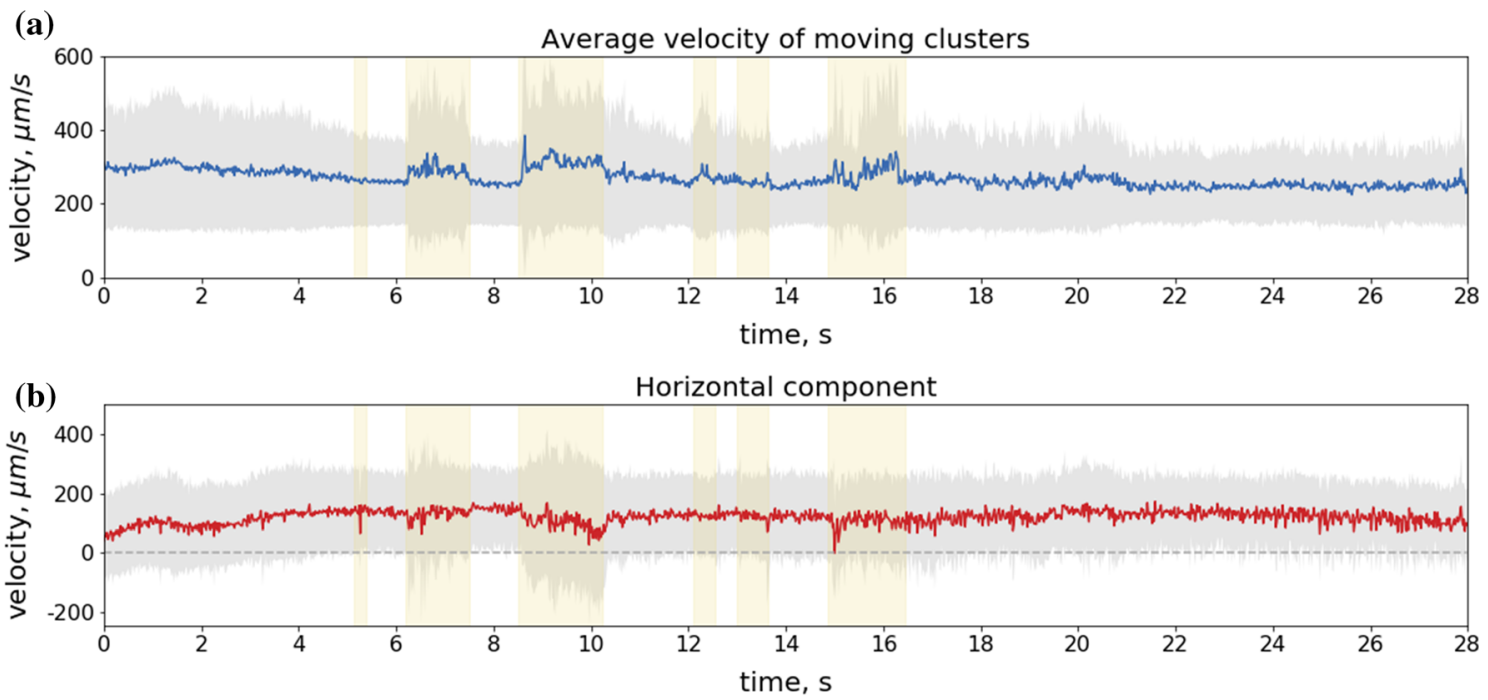

(c)

Vertical component

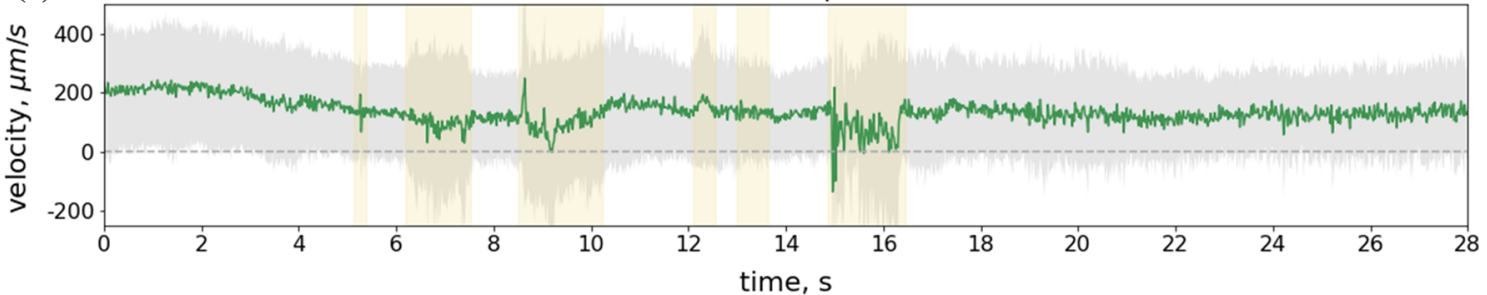

Figure 4 a Averaged absolute values of velocity vectors of all the moving clusters in a $\mathrm{AlSi} 9 \mathrm{Mg} 0.6 / \mathrm{SiC} / 20 \mathrm{p}$ film pulled in a 1500 ppm $\mathrm{O}_{2}$ atmosphere corresponding to Fig. 2, b horizontal and c vertical velocity component. The grey regions represent standard deviation. Yellow shaded regions highlight where particle motion shows changes due to specific events explained in Fig. 10.
Figure 5 a Film thickness vs. time and $\mathbf{b}$ vertical velocity vs. film thickness calculated assuming Hagen-Poiseuille flow and using 2 values for melt viscosity, namely $3.3 \mathrm{mPa}$ s and $150 \mathrm{mPa}$. (a)

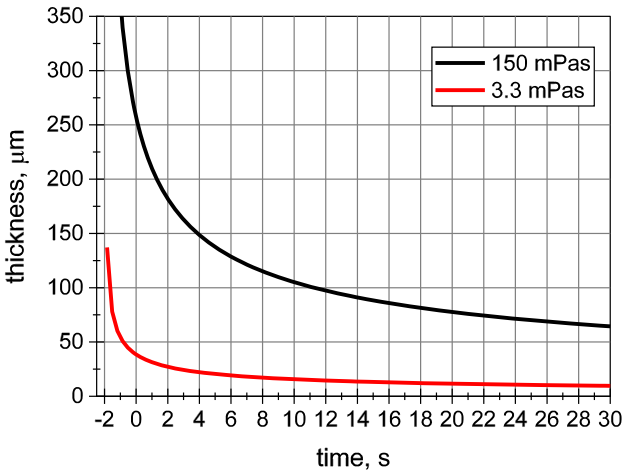

(b)

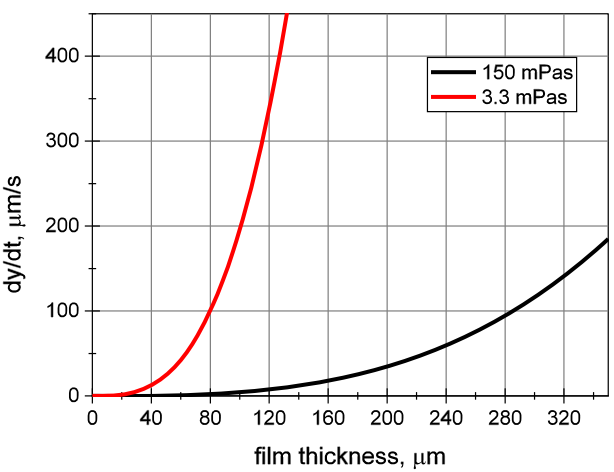

solidified lamella in this work after $x(>30 \mathrm{~s}) \approx$ $300 \mu \mathrm{m}$ as shown in Fig. 1. A possible interpretation of this result is that the model underestimates the viscosity of the liquid in the film, especially when the film thickness approached the size of the largest clusters. Applying the increased effective viscosity reported previously [23], namely $\mu=150 \mathrm{mPa}$ s for AlSi9/SiC $/ 20 \mathrm{p}$ at $700{ }^{\circ} \mathrm{C}$, the film thickness at $t=0 \mathrm{~s}$ changes to $x(0 \mathrm{~s})=250 \mu \mathrm{m}$. The time at which the horizontal velocity is zero is $t=-0.5 \mathrm{~s}$ as derived by extrapolating Fig. 4b. Evaluating Fig. 5a, we then obtain $x(-0.5 \mathrm{~s})=300 \mu \mathrm{m}$ for the initial thickness of the film.

In our experiment, the vertical velocity, and therefore the film thickness, seem to remain constant during the whole measurement time due to the stabilising effect of the $\mathrm{SiC}$ particle clusters preventing the film from further thinning. This is because there is 
sufficient liquid stored in the Plateau border that feeds the volume of the much thinner film and maintains an equilibrium during the measurement. The vertical velocity of the particle clusters corresponds to $\mathrm{d} y / \mathrm{d} t=117 \mu \mathrm{m} \mathrm{s}^{-1}$ according to Eq. (5), see Fig. $5 b$, which is quite close to the measured mean velocity of $134 \pm 41 \mu \mathrm{m} \mathrm{s}^{-1}$ given in Fig. 4c.

In Fig. 6, the velocity of particle clusters is plotted separately for three distinct particle size categories. The velocity of the largest clusters $\left(>3645 \mu \mathrm{m}^{2}\right)$ shows large statistical scatter due to their low number. Their average velocity is $\sim 400 \mu \mathrm{m} \mathrm{s}^{-1}$ at the beginning and remains higher $\left(\sim 351 \pm 63 \mu \mathrm{m} \mathrm{s}^{-1}\right)$ than the nearly constant velocities of medium-sized $\left(264 \pm 9 \mu \mathrm{m} \mathrm{s}^{-1}\right)$ and small clusters $\left(233.79 \pm 12 \mu \mathrm{m} \mathrm{s}^{-1}\right)$ in the period $0-20 \mathrm{~s}$. A notable deceleration of large clusters down to $159 \pm 62 \mu \mathrm{m} \mathrm{s}^{-1}$ takes place from $20-28 \mathrm{~s}, 30 \%$ to $40 \%$ lower than medium-sized and small particles. This effect can be explained by particle jamming caused by progressive particle attachment to surface oxide layers. Assuming spherical clusters, the diameter of the largest cluster $\left(>3645 \mu \mathrm{m}^{2}\right)$ is $D>64 \mu \mathrm{m}$, hindering the film to further thinning. This is in accordance with the observed minimal film thickness of $x_{\min } \approx 50 \mu \mathrm{m}[4,20,21]$ in metal foams. Thus, big clusters with diameters close to the film thickness might be decelerated by the parabolic velocity profile over the film thickness as postulated by Hagen-Poiseuille. This effect may be caused by friction with the surface oxide skin or with other clusters of type 2, while smaller clusters continue flowing without such hindrance through regions closer to the centre of the film. The presence of an immobilised surface skin is plausible as the gas-liquid interface oxidises immediately and forms a solid oxide skin [7, 24-26].

\section{Direction change of particle motion}

The directions of particle motions and how they change over time are specified in Fig. 7 . Both the number of particles per angle (Fig. 7a) and the total area of all particles moving in an angle interval (Fig. $7 b$ ) are given. In accordance with Fig. 3, the majority of the particles (hence in red) initially move at an angle of $180^{\circ}$, which is defined as the downward direction, i.e. they are driven by gravity. After a holding time $>7 \mathrm{~s}$, however, Fig. $7 \mathrm{a}, \mathrm{b}$ shows that some clusters undergo a gradual change in direction from vertical towards a flow to the right $\left(90^{\circ}\right)$ and into the Plateau border, compare Fig. 2. An increased number of clusters is found to move at angles around $60^{\circ}$ at specific times (upper graph), which implies that mainly smaller clusters (as most clearly seen in the video, Online Resource (1)) have an upward velocity component, i.e. opposing gravity, see also Fig. 2. In this particular case, capillary suction induced by film thinning is stronger than gravityinduced drainage. In two special time intervals, 6.2-7.6 s and 15.4-16.6 s, clusters are found to move straight upwards, but this is caused by the capture of particles by small bubbles, which appeared during stirring or film pulling. The origin of these bubbles can be gas trapped in the slag or in porous oxide skins, which can form already by $1500 \mathrm{ppm}$ of $\mathrm{O}_{2}$ [8].

An experiment on an AlSi9Mg0.6/SiC/20p film drawn in air $\left(21 \% \mathrm{O}_{2}\right)$ is represented by Fig. 8 (see videos in Online Resources 1 and 2). A high oxygen content is known to influence particle flow and

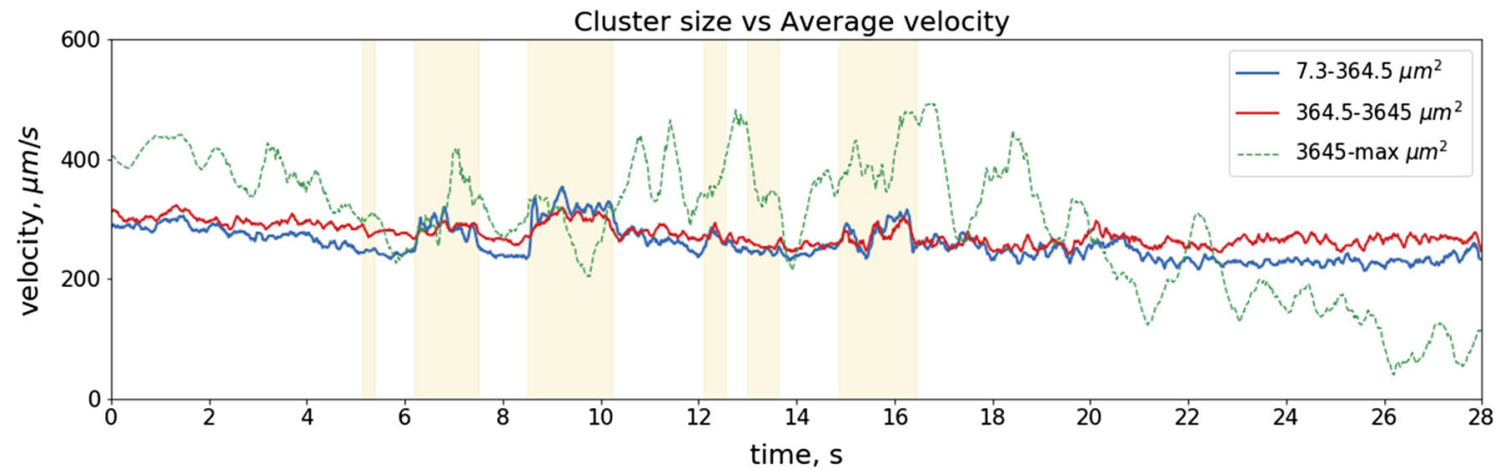

Figure 6 Average velocity of moving particle clusters in a AlSi9Mg0.6/SiC/20p film pulled in an atmosphere of $1500 \mathrm{ppm}$ $\mathrm{O}_{2}$ belonging to one of three size categories: $7.3-364.5 \mu \mathrm{m}^{2}$,
$364.5-3645 \mu \mathrm{m}^{2}$ and larger than $364.5 \mu \mathrm{m}^{2}$. Yellow shaded regions highlight where the particle motion shows changes due to specific events explained in Fig. 10. 
Figure 7 Analysis of the flow direction of particles in an AlSi9Mg0.6/SiC/20p film drawn in a 1500 ppm $\mathrm{O}_{2}$ atmosphere. The directions are specified as follows: $0^{\circ} \%$ $360^{\circ}=$ upwards, $90^{\circ}$ degrees $=$ to the right, $180^{\circ}=$ downwards, $270^{\circ}=$ to the left. Light green shaded regions highlight particle motion changes caused by specific events as explained in Fig. 10. a Number of particles that flow in a given direction at a given time and $\mathbf{b}$ total area of particles flowing in a given direction at a given time. (a)

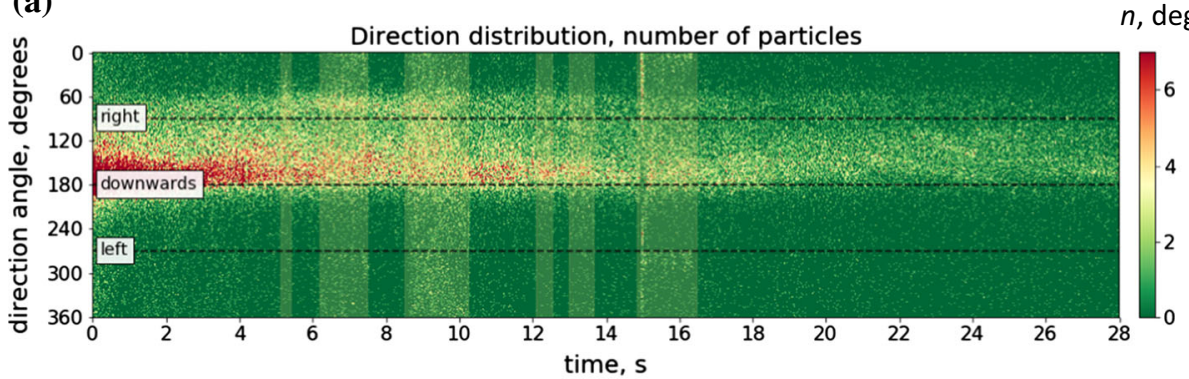

(b)

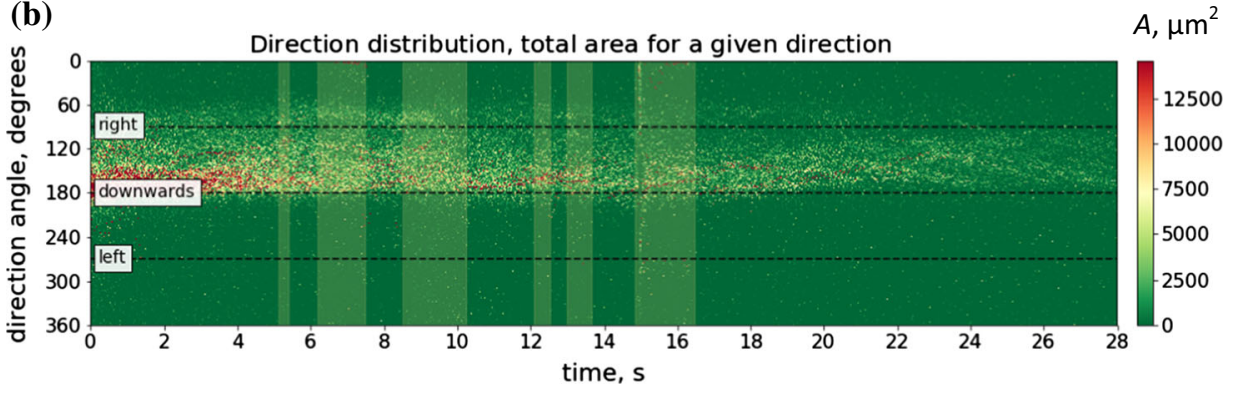

especially sticking [7], but it is not the purpose of this paper to study this in detail. A similar change of direction due to capillary suction as in the previous examples is found. This force caused by film curvature and surface tension leads to film thinning. Initially (0-4 s), particles and clusters follow curvy (vertical as well as horizontal) paths from the top of the film out of the FOV. After $6 \mathrm{~s}$, they are found to tend towards the Plateau border. In region 2 of Fig. 8b, a transition from vertical to horizontal flow into the Plateau border can be seen, which eventually predominates in (region 3), while in the Plateau

(a)

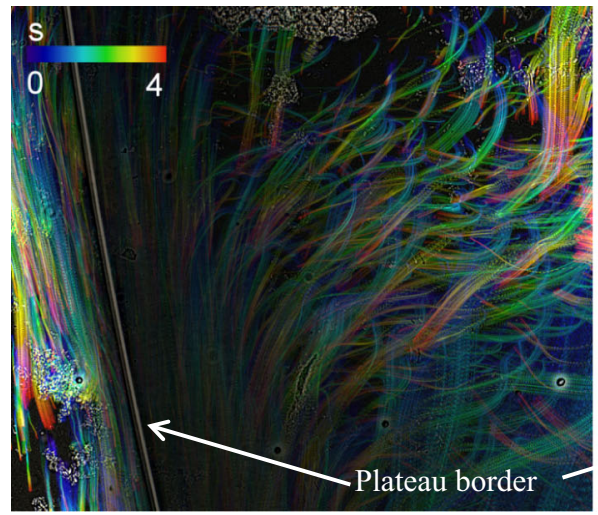

Figure $8 \mathrm{SiC}$ particle trajectories in a $\mathrm{AlSi} 9 \mathrm{Mg} 0.6 / \mathrm{SiC} / 20 \mathrm{p}$ film pulled in air from a melt at $680{ }^{\circ} \mathrm{C}$. This visualisation was obtained by image processing, particle tracking and temporal integration over 4 and $12 \mathrm{~s}$. The colour scale defines particle positions at a given time (in seconds) shown in the period a $0-4 \mathrm{~s}$ and $\mathbf{b} 6-18 \mathrm{~s}$ border vertical flow caused by gravity continues (region 1). In region 4, capillary suction is found to be strong enough to force particles to flow to the left into the Plateau border, but also upwards against gravity. Furthermore, it is found that all particles flowing into the Plateau border (velocity $\sim 100 \mu \mathrm{m} / \mathrm{s}$ ) change direction and accelerate again in region 1 (to $\sim 300$ $\mu \mathrm{m} / \mathrm{s})$. A theoretical approach was developed by Gergely and Clyne [4]. They described drainage dynamics in metal foams and found that high foam porosity, small bubbles and a vertical gradient in capillary pressure facilitate gravity-driven melt flow.

(b)

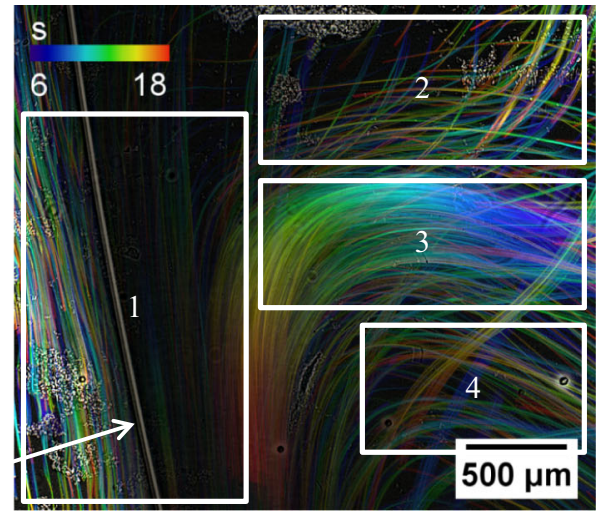

after pulling and the incipient capillary suction. (1) Vertical flow through the Plateau border. (2) Transition of vertical to horizontal flow towards the Plateau border. (3) Primarily horizontal. (4) Combination of horizontal and upwards flow. Most particles flow all through the experiment and are therefore of type 1 . 
What happens if the main exit path of particles out of a Plateau border is blocked is shown in Fig. 9. Here, particles also flow mainly downward in the first seconds but also turbulently before leaving the film or getting blocked by already fixed clusters (white noise spots). This behaviour is found to be enhanced in films pulled under $21 \% \mathrm{O}_{2}$ compared to films prepared in $1500 \mathrm{ppm}$ oxygen.

\section{Particle interactions}

By considering only raw image sequences as in Ref. [7], it was found that some initially moving particles or clusters suddenly stop and do not move any further (called type 3 ). The reason was found in interactions with the growing oxide layer on the film surfaces [7].

In the current paper, we find that such clusters do not necessarily remain trapped indefinitely, but are able to detach and attach again (DA process). To visualise this effect, Fig. 10 shows the results of several image processing steps such as background correction and integration over $0.1 \mathrm{~s}$ of all pixels above a given minimum intensity threshold. They emphasise particles in motion (type 1 and 3 partially). To highlight only particles of type 3 (green), particles that remain fixed throughout the experiment (type 2) are eliminated by subtraction of the average of the first $1 \mathrm{~s}$, whereas particles of type 1 are still visible (grey) by blurring particle trajectories obtained by integration. In Fig. 10, fast moving particles (type 1 and 3 before attachment) are marked in dark grey, (a)

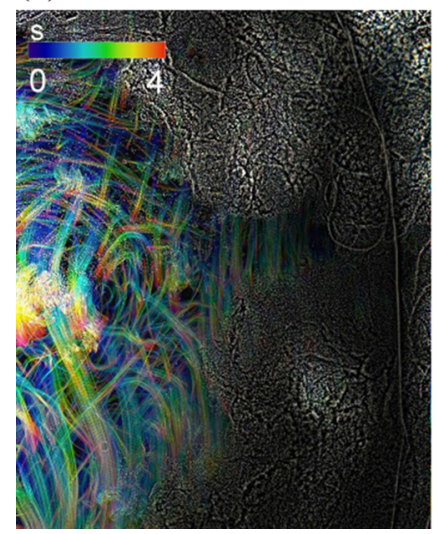

(b)

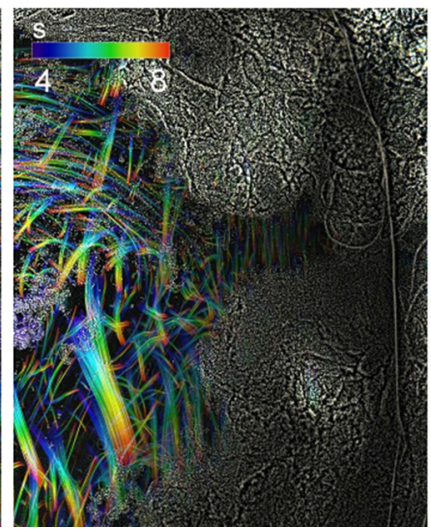

whereas particles which attach and might get Here it was found that this phenomenon is linked closely to the liquid dynamics already presented in previous figures. Thus, each column in Fig. 10 represents one specific event of Figs. 3, 4, 5, 6, 7 (yellow bars). The first event-after $5.2 \mathrm{~s}$-shows, that an already fixed cluster (type 3) undergoes a DA process, although the motion is small and difficult to observe. Considering Fig. 4, it can also be seen that the flow of this cluster is rather wiggling than directed straight downwards. After $6.2 \mathrm{~s}$, a particle cluster entering the FOV from the bottom rises and attaches to an already fixed cluster (Fig. 10b). This fast upwards flow is driven by the buoyancy of the attached gas bubble and can also be found in Fig. 7 . Although the flow is upwards, those particle-bubbleclusters can also be fixed (7.6-7.7 s). The event in the third column (after $8.5 \mathrm{~s}$, Fig. 10c) is similar to the first, where the particle cluster detaches but leaves the FOV and does not attach again. Furthermore, this cluster is mostly an agglomeration of several small particles as it can be seen in comparison with Fig. 3 . Its slight trend to the Plateau border is caused by capillary suction. After $12.3 \mathrm{~s}$ and $13.1 \mathrm{~s}$ (Fig. 10d), two further DA processes of the same cluster as in Fig. 10a are shown. The special behaviour between $12.3-12.6 \mathrm{~s}$ is that particles are wiggling and only moving slightly downward, whereas between 13.1 and $13.7 \mathrm{~s}$ (Fig. 10e) the DA process is more dominating, so that three neighbouring clusters are affected at the same time. In the last column, Fig. 10f, the released again (type 3 only) are marked in green.
Figure 9 Trajectories of continuously moving $\mathrm{SiC}$ particles (i.e. type 1) in an AlSi9Mg0.6/SiC/20p film pulled in air from a melt at $680^{\circ} \mathrm{C}$. This visualisation was obtained by image processing, particle tracking and temporal integration over $4 \mathrm{~s}$ each. The (c)

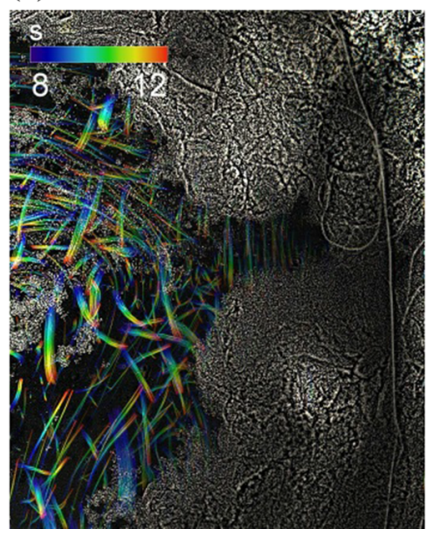

(d)

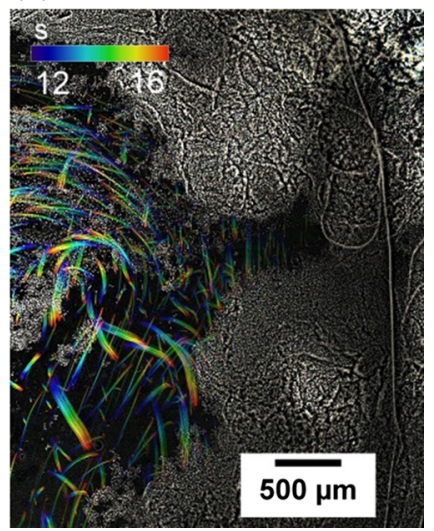

colour scale defines particle positions at a given time in the intervals a $0-4 \mathrm{~s}, \mathrm{~b} 4-8 \mathrm{~s}, \mathrm{c}$ 8-12 $\mathrm{s}$ and $\mathbf{d} 12-16 \mathrm{~s}$ and shows turbulences caused by a blocked Plateau border (right). 
(a)

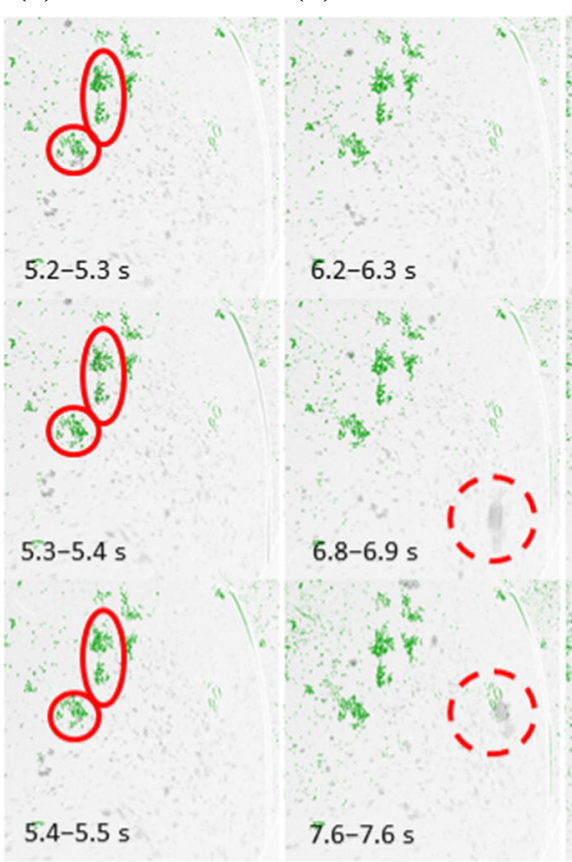

(c)

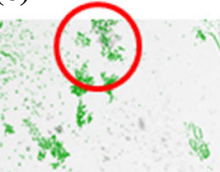

$8.5-8.6 \mathrm{~s}$

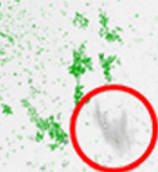

$9.5-9.6 \mathrm{~s}$

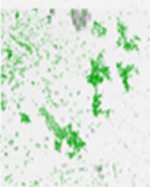

9.9-10

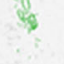

Figure 10 Integrated (over $0.1 \mathrm{~s}$ ) and image processed X-ray radioscopies of moving particles (type $1+3$ ) in an AlSi9Mg0.6/ $\mathrm{SiC} / 20 \mathrm{p}$ film pulled in $1500 \mathrm{ppm} \mathrm{O}_{2}$ at $680{ }^{\circ} \mathrm{C}$, the same experiment as in Fig. 2. Each column represents one of the yellow shaded areas of Figs. 3, 4, 5, 6, 7. Dark grey spots indicate

global change is even more conspicuous: after $15.1 \mathrm{~s}$ seven already fixed clusters detach, whereas six flow down and one upwards. The reason for the upwards flow is the same as in the second column and caused by attached gas bubbles. Triggering of this collective movement seems to be spontaneous, as the global velocity (Fig. 4) or direction distribution (Fig. 7) is constant for time periods $<15.1 \mathrm{~s}$. One suggestion is that the surface oxide layer ruptures perpendicular to the pulling direction as the curvature, respectively surface, of the film evolves during thinning [10]. Fixed particles can move together with the oxide layer until the new oxide layer forms and the film surface rests again. This is reasonable as $\mathrm{Al}$ is known to oxidise immediately [27]. This effect should be even larger in a film that is part of an expanding metal foam and that is stretched continuously unlike the more static film studied here.

\section{Conclusions}

- The dynamics of solid SiC particles in individual liquid aluminium alloy films are visualised by upwards. gravity. $20 \mathrm{~s}$. (d)

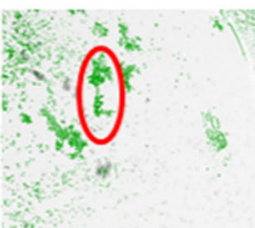

$12.3-12.4 \mathrm{~s}$

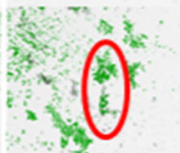

(e)

(f)
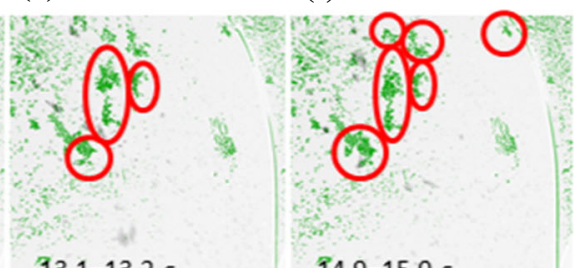

13.1-13.2

$14.9-15.0 \leq$
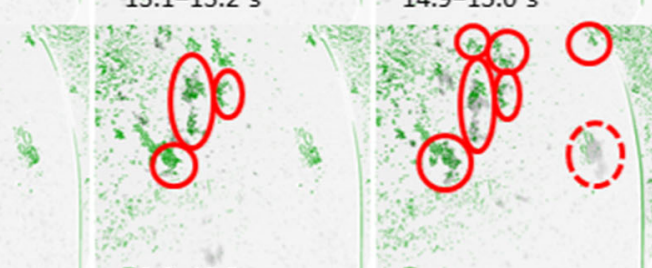

$12.5-12.6 \mathrm{~s}$

$13.2-13.3 \mathrm{~s}$

15.1-15.2s

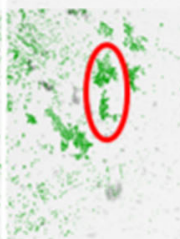

$12.8-12.9 \mathrm{~s}$
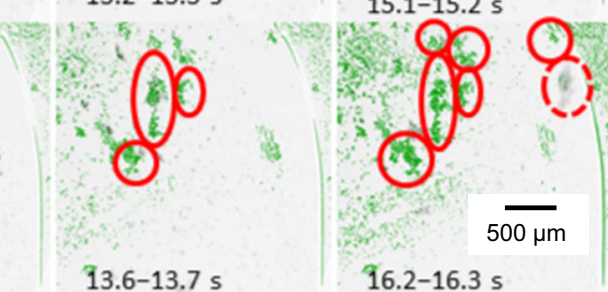

$16.2-16.3 \mathrm{~s}$

moving particles or particle clusters, green spots immobile particles or clusters. Red circles highlight already fixed particles which detach later (turn grey), flow down and might attach again (turn green again). The dashed red circles indicate flowing particles

synchrotron X-ray radioscopy and their number, agglomeration level, motion path and velocity analysed by novel image processing techniques.

- Particles in the liquid alloy can move alone but usually travel as clusters. Initially, they move mainly downwards driven by gravity, but can change their direction and move sideward to a neighbouring Plateau border due to capillary suction. In some cases, particles even flow upward because capillary forces dominate over

- The moving particles are divided into 3 categories, namely: permanently fixed clusters (type 2), clusters which were moveable but become trapped at a certain instant (type 3) and continuously moving particles (type 1).

- Big clusters' velocity differs from the velocity of medium and small ones, indicating that in our experiments their flow starts being hindered after

- A constant film thickness of about $300 \mu \mathrm{m}$ is established due to the stabilising action of the $\mathrm{SiC}$ particles a few seconds after pulling the film. This 
leads to the almost constant vertical flow velocity during our experiment.

- Sudden changes in the velocity plots point at specific events, e.g. a detachment-attachment process of fixed particles or clusters.

- A collective movement of previously fixed particles was found and shown as sudden changes in the velocity plots, which can be explained as ruptures in the film oxide skin.

- Clusters can be trapped either by the oxide layers or by other immobilised clusters.

\section{Acknowledgement}

Open Access funding provided by Projekt DEAL. This study was supported by the Deutsche Forschungsgemeinschaft, grants GA 1304/2-1, BA 1170/17-1, GA 1304/5-1 and BA 1170/35-1. We acknowledge the ESRF for providing synchrotron radiation beam at the ID19 beamline through proposal MA-1627.

\section{Compliance with ethical standards}

Conflict of interest There are no conflicts to declare.

Electronic supplementary material: The online version of this article (https://doi.org/10.1007/s108 53-020-05007-5) contains supplementary material, which is available to authorized users.

Open Access This article is licensed under a Creative Commons Attribution 4.0 International License, which permits use, sharing, adaptation, distribution and reproduction in any medium or format, as long as you give appropriate credit to the original author(s) and the source, provide a link to the Creative Commons licence, and indicate if changes were made. The images or other third party material in this article are included in the article's Creative Commons licence, unless indicated otherwise in a credit line to the material. If material is not included in the article's Creative Commons licence and your intended use is not permitted by statutory regulation or exceeds the permitted use, you will need to obtain permission directly from the copyright holder. To view a copy of this licence, visit http://creativecommons.org/licen ses/by/4.0/.
Electronic supplementary material: The online version of this article (https://doi.org/10.1007/s108 53-020-05007-5) contains supplementary material, which is available to authorized users.

\section{References}

[1] Weaire D, Hutzler S (1999) The physics of foams. Oxford University Press, Oxford

[2] Gergely V, Clyne B (2000) The FORMGRIP process: foaming of reinforced metals by gas release in precursors. Adv Eng Mater 2(4):175-178

[3] Heim K, García-Moreno F, Banhart J (2018) Particle size and fraction required to stabilise aluminium alloy foams created by gas injection. Scripta Mater 153:54-58

[4] Gergely V, Clyne TW (2004) Drainage in standing liquid metal foams: modelling and experimental observations. Acta Mater 52(10):3047-3058

[5] Babcsán N, Leitlmeier D, Banhart J (2005) Metal foamshigh temperature colloids: part I. Ex situ analysis of metal foams. Colloids Surf A 261(1-3):123-130

[6] Haibel A, Rack A, Banhart J (2006) Why are metal foams stable? Appl Phys Lett 89:154102

[7] Heim K, Vinod-Kumar GS, García-Moreno F, Rack A, Banhart J (2015) Stabilisation of aluminium foams and films by the joint action of dispersed particles and oxide films. Acta Mater 99:313-324

[8] Heim K, Kumar GSV, Garcia-Moreno F, Banhart J (2014) Role of ambient oxygen in the stabilisation of single aluminium alloy films. Proc Mater Sci 4:263-268

[9] Heim K, Garcia-Moreno F, Vinod Kumar GS, Rack A, Banhart J (2014) The rupture of a single liquid aluminium alloy film. Soft Matter 10(26):4711-4716

[10] Vinod Kumar GS, Garcia-Moreno F, Babcsan N, Brothers AH, Murty BS, Banhart J (2007) Study on aluminium-based single films. Phys Chem Chem Phys 9(48):6415-6425

[11] Heim K, Kumar GSV, Garcia-Moreno F, Manke I, Banhart J (2013) Drainage of particle-stabilised aluminium composites through single films and Plateau borders. Colloids Surf, A 438:85-92

[12] Garcia-Moreno F, Rack A, Helfen L, Baumbach T, Zabler S, Babcsan N, Banhart J, Martin T, Ponchut C, Di Michiel M (2008) Fast processes in liquid metal foams investigated by high-speed synchrotron x-ray microradioscopy. Appl Phys Lett 92(13):3

[13] Schneider CA, Rasband WS, Eliceiri KW (2012) NIH Image to ImageJ: 25 years of image analysis. Nat Methods 9:671-675 
[14] Zabler S, Ershov A, Rack A, Garcia-Moreno F, Baumbach T, Banhart J (2013) Particle and liquid motion in semi-solid aluminium alloys: A quantitative in situ microradioscopy study. Acta Mater 61(4):1244-1253

[15] Myagotin A, Ershov A, Helfen L, Verdejo R, Belyaev A, Baumbach T (2012) Coalescence analysis for evolving foams via optical flow computation on projection image sequences. J Synchrotron Rad 19(4):483-491

[16] Fisher LR, Mitchell EE, Hewitt D, Ralston J, Wolfe J (1991) The drainage of a thin aqueous film between a solid surface and an approaching gas bubble. Colloids Surf 52:163-174

[17] Brady AP, Ross S (1944) The measurement of foam stability. J Am Chem Soc 66(8):1348-1356

[18] Laimböck PR (1998) Foaming of glass melts. PhD, Technische Universiteit Eindhoven, Eindhoven

[19] Gebhardt E, Detering K (1959) Über die Eigenschaften metallischer Schmelzen: XVI. Die innere Reibung eutektischer Aluminiumlegierungen. Z Metallkd 50(7):379-385

[20] Flemings MC (1991) Behavior of metal-alloys in the semisolid state. Metal Trans A: Phys Metal Mater Sci 22(5):957-981

[21] Deqing W, Ziyuan S (2003) Effect of ceramic particles on cell size and wall thickness of aluminum foam. Mater Sci Eng, A 361(1):45-49
[22] Babcsán N, Leitlmeier D, Degischer HP (2003) Foamability of particle reinforced aluminum melt. Materialwiss Werkstofftech 34(1):22-29

[23] Garcia-Moreno F, Tobin ST, Mukherjee M, Jimenez C, Solorzano E, Vinod Kumar GS, Hutzler S, Banhart J (2014) Analysis of liquid metal foams through X-ray radioscopy and microgravity experiments. Soft Matter 10(36):6955-6962

[24] Babcsan N, Leitlmeier D, Degischer H, Banhart J (2004) The role of oxidation in blowing particle-stabilised aluminium foams. Adv Eng Mater 6:421-428

[25] Dudka A, Garcia-Moreno F, Wanderka N, Banhart J (2008) Structure and distribution of oxides in aluminium foam. Acta Mater 56(15):3990-4001

[26] Simancik F, Behulova K, Bors L (2001) Effect of ambient atmosphere on the foam expansion. In: Banhart J, Ashby MF, Fleck NA (eds) Cellular Metals and Metal Foaming Technology: Metfoam. MIT Publishing, Bremen, pp 89-92

[27] DebRoy T, Bandopadhyay A, Roy R (1994) Oxide matrix composite by directional oxidation of a commercial aluminum-magnesium alloy. J Am Ceram Soc 77(5):1296-1300

Publisher's Note Springer Nature remains neutral with regard to jurisdictional claims in published maps and institutional affiliations. 